\title{
Long-term outcome of patients with steroid-refractory acute severe UC treated with ciclosporin or infliximab
}

\author{
D Laharie, ${ }^{1}$ A Bourreille, ${ }^{2} \mathrm{~J}$ Branche,${ }^{3} \mathrm{M}$ Allez, ${ }^{4}$ Y Bouhnik, ${ }^{5} \mathrm{~J}$ Filippi, ${ }^{6}$ F Zerbib, ${ }^{1} \mathrm{G}$ Savoye,${ }^{7}$ L Vuitton, ${ }^{8} \mathrm{~J}$ \\ Moreau, ${ }^{9}$ A Amiot, ${ }^{10} \mathrm{~J}$ Cosnes, ${ }^{11}$ E Ricart, ${ }^{12} 0$ Dewit, ${ }^{13}$ A Lopez-Sanroman, ${ }^{14}$ M Furriery, ${ }^{15}$ F Carbonnel, ${ }^{16} \mathrm{G}$ \\ Bommelaer, ${ }^{17}$ B Coffin, ${ }^{18}$ X Roblin, ${ }^{19} \mathrm{G}$ van Assche, ${ }^{20} \mathrm{M}$ Esteve, ${ }^{21} \mathrm{M}$ Farkkila, ${ }^{22} \mathrm{JP}$ Gisbert, ${ }^{23} \mathrm{P}$ Marteau, ${ }^{11} \mathrm{~S}$ \\ Nahon, ${ }^{24} \mathrm{M}$ de Vos, ${ }^{25} \mathrm{~J}$ Lambert, ${ }^{26} \mathrm{JY}$ Mary, ${ }^{26} \mathrm{E}$ Louis, ${ }^{27}$ for the Groupe d'Etudes Thérapeutiques des Affections \\ Inflammatoires Digestives
}

${ }^{l}$ CHU de Bordeaux, Hôpital Haut-Lévêgue, Service d'Hépato-gastroentérologie et oncologie digestive-Universite de Bordeaux, Bordeaux, France

${ }^{2}$ CHU de Nantes, Hôtel-Dieu, Hépato-Gastroentérologie, Institut des Maladies de l'Appareil Digestif, Nantes, France

${ }^{3}$ CHRU de Lille, Hôpital Claude Huriez, Service des maladies de l'appareil digestif-Endoscopie digestive, Lille, France

${ }^{4}$ Hôpital Saint-Louis, service d'Hépato-Gastroentérologie, APHP-Université Paris VII, Paris, France

${ }^{5}$ Hôpital Beaujon, Gastroentérologie, MICI et Assistance Nutritive, APHP-Université Paris VII, Clichy, France

${ }^{6} \mathrm{CHU}$ de Nice, Hôpital de l'Archet 2, Service de Gastroentérologie et Nutrition Clinigue, Nice, France

${ }^{7} \mathrm{CHU}$ de Rouen, Hôpital Charles Nicolle, service de Gastroentérologie, UMR 1073", Normandie Université-Rouen, Rouen, France

${ }^{8} \mathrm{CHU}$ de Besançon, Hôpital Jean Minjoz, Service de Gastroentérologie, Besançon, France

${ }^{9} \mathrm{CHU}$ de Toulouse, Hôpital Rangueil, Service de Gastro-entérologie et Nutrition, Toulouse, France

${ }^{10}$ Hôpital Henri Mondor, Service d'Hépato-gastroentérologie, APHP-Université Créteil, Créteil, France

${ }^{11}$ Hôpital St-Antoine, service de Gastroentérologie, Paris, France

${ }^{12}$ Gastroenterology Department, Hospital. Clinic, IDIBAPS, CIBEREHD, Barcelona, Spain

${ }^{13}$ UCL Saint Luc, Service d'Hépato-Gastroentérologie, Brussels, Belgium

${ }^{14}$ Hospital Ramon y Cajal, Unidad de Ell/lBD Unit, Servicio de Gastroenterología y Hepatología, Madrid, Spain

${ }^{15}$ CHU Amiens, Hôpital Nord, service d'Hépato-Gastroentérologie, Amiens, France

${ }^{16}$ Hôpital Bicêtre, service d'Hépato-Gastroentérologie, APHP_Université Paris Sud 11, Le Kremlin Bicêtre, France

${ }^{17} \mathrm{CHU}$ Clermont-Ferrand, Service Hépatologie-Gastro-entérologie, Clermont-Ferrand, France

${ }^{18}$ Hôpital Louis Mourier, service d'Hépato-Gastroentérologie, Pôle Maladie Appareil Digestif, APHP-Université Paris VII, Colombes,

France

${ }^{19} \mathrm{CHU}$ de Saint-Etienne, Hôpital Nord, Service de Gastro-entérologie et Hépatologie, Saint-Etienne, France

${ }^{20}$ Division of Gastroenterology, University Hospital of Leuven, Leuven, Belgium

${ }^{21}$ Department of Gastroenterology, Hospital Universitari Mútua de Terrassa, University of Barcelona, Terrassa. CIBEREHD, Catalonia,

${ }^{22}$ Helsinki University, and Helsinki University Central Hospital, Clinic of Gastroenterology, HUS, Finland

${ }^{23}$ Gastroenterology Unit, Hospital Universitario de La Princesa, Institute de Investigación Sanitaria Princesa (IIS-IP) y Centra de Investigación Biomedica en Red de Enfermedades Hepáticas y Digestivas (CIBEREHD), Madrid, Spain

${ }^{24}$ CHI Le Raincy Montfermeil, Service d'Hépato-gastroentérologie, Montfermeil, France

${ }^{25}$ Ghent University Hospital, Gent, Belgium

${ }^{26}$ UMR-S- 1153 Inserm, Eguipe ECSTRA, Denis Diderot-Paris 7 University, Hôpital Saint-Louis, Paris, France

${ }^{27}$ Department of Gastroenterology, University Hospital CHU of Liège, Liège, Belgium

\section{ABSTRACT}

Objective Ciclosporin and infliximab have demonstrated short-term similar efficacy as second-line therapies in patients with acute severe UC (ASUC) refractory to intravenous steroids. The aim of this study was to assess long-term outcome of patients included in a randomised trial comparing ciclosporin and infliximab. Design Between 2007 and 2010, 115 patients with steroid-refractory ASUC were randomised in 29 European centres to receive ciclosporin or infliximab in association with azathioprine. Patients were followed until death or last news up to January 2015. Colectomy-free survival rates at 1 and 5 years and changes in therapy were estimated through Kaplan-Meier method and compared between initial treatment groups through log-rank test. Results After a median follow-up of 5.4 years, colectomy-free survival rates $(95 \% \mathrm{CI})$ at 1 and 5 years were, respectively, $70.9 \%(59.2 \%$ to $82.6 \%)$ and $61.5 \%(48.7 \%$ to $74.2 \%)$ in patients who received ciclosporin and $69.1 \%(56.9 \%$ to $81.3 \%$ ) and $65.1 \%(52.4 \%$ to $77.8 \%)$ in those who received infliximab (p=0.97). Cumulative incidence of first infliximab use at 1 and 5 years in patients initially treated with ciclosporin was, respectively, $45.7 \%(32.6 \%$ to $57.9 \%)$ and $57.1 \%$ (43.0\% to $69.0 \%)$. Only four patients from the infliximab group were subsequently switched to ciclosporin. Three patients died during the follow-up, none directly related to UC or its treatment. Conclusions In this cohort of patients with steroid-refractory ASUC initially treated by ciclosporin or infliximab, long-term colectomy-free survival was independent from initial treatment. These long-term results further confirm a similar efficacy and good safety profiles of both drugs and do not favour one drug over the other. 
Trial registration number EudraCT: 2006-005299-42; ClinicalTrials.gouv number: NCT00542152; postresults.

\section{Significance of this study}

\section{What is already known on this subject?}

In patients with acute severe UC refractory to intravenous steroids, ciclosporin and infliximab are efficient second-line therapies to avoid emergent colectomy.

- Both drugs have similar short-term efficacy and good safety profile.

- Few long-term data comparing both drugs are available so far.

\section{What are the new findings?}

- Long-term colectomy-free survival is independent from initial second-line treatment.

- Higher proportion of patients initially treated with ciclosporin needed a new treatment than those who received infliximab first.

- Nearly half of patients firstly treated with ciclosporin will switch within 1 year to infliximab.

- Bridge therapy with ciclosporin to thiopurine was successful on long term in half of patients.

\section{How might it impact on clinical practice in the foreseeable future?}

- Whatever the drug started as rescue therapy in patients with steroid-refractory acute severe UC, long-term colectomy rates are similar.

- Choice between both drugs should not only be guided by efficacy and safety criteria that are close.

- Costs, administration route and patient's preference should also be taken into account in daily practice.

\section{INTRODUCTION}

UC is a lifelong IBD that could be complicated by acute severe flare in $20 \%-25 \%$ of patients. $^{12}$ Nowadays, acute severe UC (ASUC) remains a life-threatening condition, with 1\%-2\% death rate in Western countries $^{34}$ requiring an emergent management in specialised units.

Most recent guidelines recommend using Truelove-Witts criteria to identify patients with ASUC who should receive high doses of intravenous steroids at admission. ${ }^{56}$ However, approximately $40 \%$ of patients do not respond adequately to this regimen. ${ }^{6}$ To avoid colectomy, controlled trials have demonstrated the efficacy of both ciclosporin, a calcineurine inhibitor, and infliximab, an anti-tumor necrosis factor (TNF) monoclonal antibody, as second-line medical therapy ${ }^{5-9}$

Two trials have directly compared ciclosporin and infliximab in patients with steroid-refractory ASUC. A European open-label randomised study (CYSIF) including 115 patients could not demonstrate any superiority of intravenous ciclosporin over a standard induction infliximab regimen. ${ }^{10}$ More recently, in a comparative pragmatic trial (CONSTRUCT) that enrolled 270 patients in UK, the quality-adjusted survival over 12-36 months was similar to both agents. ${ }^{11}$ Overall, ciclosporin and infliximab provided high rates of early clinical response, over $80 \%$ within the first week, with acceptable safety profiles.

Little is known about the long-term outcome of ASUC in patients receiving such second-line medical therapies and few long-term data comparing both drugs are available so far. The aim of this study was to assess long-term outcome of patients included in the CYSIF trial.

\section{PATIENTS AND METHODS}

\section{Initial study design and patients}

CYSIF was a randomised, open-label, 98-day controlled trial conducted from June 2007 to August 2010 in 23 French and Belgian GETAID (Groupe d'Etude sur les Affections Inflammatoires Digestives) and 6 European 
ECCO (European Crohn and Colitis Organisation) centres. The institutional review board at each centre approved the protocol, and all patients provided written informed consent. Characteristics of the 115 patients included and results of the initial study have been previously published in extenso. ${ }^{10}$

Eligible patients were adults admitted for ASUC defined by a Lichtiger score $>10$, who were refractory to highdose intravenous steroid therapy (at least $0.8 \mathrm{mg} / \mathrm{kg} / \mathrm{day}$ of methylprednisolone or equivalent) given for at least 5 days. They were naive for ciclosporin, infliximab and thiopurine except if it was started $<4$ weeks before inclusion. Patients with indication for emergent colectomy, proctitis, Crohn's disease, active infection or usual contraindication to ciclosporin, infliximab and thiopurine were excluded.

To summarise the study design, at day 0 (inclusion visit) randomisation assigned patients to receive ciclosporin, administered by continuous intravenous infusion at the initial dose regimen of $2 \mathrm{mg} / \mathrm{kg} / \mathrm{day}$, or infliximab at the dose of $5 \mathrm{mg} / \mathrm{kg}$. Treatments were not blinded to patients and investigators. In patients from both groups with clinical response at day 7, steroids were switched orally and rapidly tapered according to a standardised regimen, and azathioprine was started at $2-2.5 \mathrm{mg} / \mathrm{kg} / \mathrm{day}$ or continued for patients still on. In the same time and according to the randomisation arm, intravenous ciclosporin was switched orally or two additional infliximab infusions at the same dose were administered at days 14 and 42. Primary endpoint of the trial was treatment failure at any time, defined by the presence of at least one of the following criteria: absence of clinical response at day 7 , relapse between days 7 and 98, absence of steroid-free remission at day 98 or severe adverse event leading to treatment interruption, colectomy or death. ${ }^{10}$

Study period ended at day 98 for patients who completed the trial. Since the end of the study, patients were treated according to clinical need as judged by their treating physician. Drugs used were those usually employed in UC, according to licensed or published doses and frequency.

\section{Data update}

Data from patients included in the initial study were updated retrospectively through a standardised form from day 98 for patients who completed the study period and from date of early termination for the others. Regimen and duration of treatments received since inclusion (ciclosporin or infliximab and azathioprine) were recorded. The following events and their date were collected: rescue therapy in case of non-primary response (defined by the absence of clinical response at day 7 or disease relapse within the study period), disease relapse in case of primary response to ciclosporin or infliximab (defined by recurrence of disease symptoms beyond the study period with a need for systemic treatment excluding 5-amino-salicylate acid (ASA) and/or topical therapies), colectomy (type of surgery, postoperative complication within 30 days and number of surgical steps) and death.

\section{Outcomes and follow-up}

Follow-up started at the time of inclusion into the CYSIF trial. The following events were studied per treatment group, as given at inclusion into the trial: (1) death; (2) colectomy; (3) first use of any additional UC systemic treatment-including steroids, ciclosporin, infliximab and azathioprine or biologics and, excluding 5-ASA; (4) first switch to infliximab for patients receiving initially ciclosporin and vice versa; (5) first use of a new systemic treatment other than those given at inclusion, allowing ciclosporin and/or azathioprine discontinuation and restart in patients receiving ciclosporin, infliximab and/or azathioprine discontinuation and restart and infliximab optimisation in those receiving infliximab. In patients who experienced an event, time-to-event was the time interval between the beginning of the follow-up and event occurrence. In patients who did not experience any event, follow-up was censored at the date of point (1 January 2015) or at the date of last news, whatever occurred first.

\section{Statistical analysis}

Patient characteristics were described through frequency (proportion) or median (IQR). In the general description of the patient follow-up, time durations were expressed as median (IQR), except in groups of four patients or less in which values or range were provided.

Cumulative incidence of first use of an additional UC systemic treatment, of first switch to infliximab for patients receiving initially ciclosporin and of first use of a new systemic treatment other than those given at inclusion which estimates the marginal probability of the event ${ }^{12}$ was calculated taking into account death or colectomy whatever occurred first as a competing event, and described through percentage and 95\% CI, number of events, number of death or colectomy and number of patients at risk at prespecified time points after inclusion 
into the trial. Cumulative incidence curves were compared between treatment groups through Gray's test and difference expressed through absolute risk regression (ARR) estimate and 95\% CI. ${ }^{13}$

Colectomy-free survival curves were derived through the Kaplan-Meier method and described through the number of events/number of patients, percentage and 95\% CI and number of patients at risk at prespecified time points after inclusion into the trial. Colectomy-free survival curves were compared between treatment groups through the log-rank test and difference expressed through HR estimate and 95\% CI.

\section{RESULTS}

\section{Study population at inclusion}

Patients were recruited for the initial study in 29 centres in France, Spain, Belgium and Finland from June 2007 to August

2010. Among the 115 randomised patients, 58 patients were assigned to receive ciclosporin and 57 to infliximab. As two patients from the infliximab arm have received ciclosporin instead of the treatment allocated by randomisation, due to patient's wish in one case and to an error of administration in the other, 60 patients actually received ciclosporin and 55 infliximab in the initial study and were considered for the present follow-up.

Baseline characteristics according to treatment received at inclusion in the initial study are shown in table 1. They were similar in both groups including endoscopic activity, except for median Lichtiger score and median $\mathrm{C}$ reactive protein (CRP) level which were significantly higher in patients who received infliximab, but median Mayo score and proportion of patients with CRP level above $50 \mathrm{mg} / \mathrm{L}$ were not.

Table 1 Demographic and clinical characteristics of the 115 patients

\begin{tabular}{lccc}
\hline Characteristic & Ciclosporin $(\mathbf{n = 6 0})$ & Infliximab $(\mathbf{n = 5 5})$ & p Value \\
\hline Female gender, $\mathrm{n}(\%)$ & $28(47)$ & $27(49)$ & 0.79 \\
Median age, years (IQR) & $39(26-50)$ & $36(26-51)$ & 0.48 \\
Median disease duration, years (IQR) & $2.5(0.5-7.3)$ & $0.8(0.1-4.4)$ & 0.07 \\
First attack of UC, $\mathrm{n}(\%)$ & $10(17)$ & $16(29)$ & 0.11 \\
Disease location E3, ${ }^{18} \mathrm{n}(\%)$ & $34(57)$ & $31(56)$ & 0.93 \\
Patient naive to azathioprine, $\mathrm{n}(\%)$ & $56(93)$ & $51(93)$ & 0.90 \\
Median intravenous, steroid duration, days (IQR) & $7(6-9) *$ & $8(6-9) \mathrm{t}$ & 0.72 \\
Median Lichtiger score (IQR) & $12(11-13)$ & $13(12-14)$ & 0.004 \\
Median Mayo disease activity index (IQR) & $10(10-11)$ & $11(10-11)$ & 0.26 \\
Mayo endoscopic subscore=3, n (\%) & $57(95)$ & $53(96)$ & 0.73 \\
Median CRP (mg/L, IQR) & $30(16-66) \dagger$ & $46(28-73) \dagger$ & 0.044 \\
Median albumin (g/L, IQR) & $28(24-32) \S$ & $27(23-33) \mathbb{I}$ & 0.55 \\
\hline
\end{tabular}

$* \mathrm{n}=59$.

$\dagger \mathrm{n}=54$.

$\$ n=57$.

$\S n=56$.

In $\mathrm{n}=49$.

UC location according to the Montreal classification ${ }^{18}(\mathrm{E} 1: \mathrm{n}=\mathrm{O})$.

$\mathrm{CRP}, \mathrm{C}$ reactive protein.

\section{Description of follow-up}

The median follow-up time was 5.4 years (IQR: 4.7-6.2).

Among the 60 patients who started ciclosporin at inclusion (figure 1A), there were 34 failures, 24 successes and 2 lost to follow-up in the initial study. All patients discontinued ciclosporin at the end of the study period and received maintenance with azathioprine alone. Among the 34 failures, 5 patients underwent directly colectomy after $0.4(0.2-0.8)$ months, 21 received rescue therapy after $1(0.3-3.2)$ month $(2$ were treated with steroids and 19 with infliximab $-5 \mathrm{mg} / \mathrm{kg}$ in all except one patient who received $10 \mathrm{mg} / \mathrm{kg}$ ) and 8 did not receive rescue 
therapy. Among the 19 patients who received infliximab rescue therapy after ciclosporin failure, 10 underwent colectomy after 3 (1-8) months (including three with dose intensification), 1 was lost to follow-up 50 months after rescue, 1 died (case detailed below) and the remaining 7 patients (including the 2 who received steroids as rescue therapy) reached end of follow-up with no new event 56 (43-71) months after rescue. Among the 8 patients with ciclosporin failure who did not receive rescue therapy, 1 reached end of follow-up 60 months after inclusion without new systemic treatment and 7 had new systemic treatment 8 (4-20) months after inclusion: 5 treated with infliximab reached end of follow-up 57 (45-67) months after treatment and 2 treated by either steroids or infliximab underwent colectomy 4 and 25 months later. Among the 24 patients who received ciclosporin successfully treated at the end of the study period, 9 had new systemic treatments 6 (4-29) months after inclusion: 2 underwent colectomy 2 and 17 months later despite infliximab rescue, and 7 received various medical therapies ( 1 infliximab, 1 steroids, 1 steroids then infliximab, 1 golimumab, 3 infliximab then adalimumab) and were still followed up 53 (41-72) months later. Among the 15 patients without new treatment, 2 died (cases detailed below) and 13 reached end of follow-up 69 (54-82) months after inclusion.

Figure 1 Flowchart of patients according to the treatment given at inclusion (ciclosporin (CsA) in A and infliximab (IFX) in B).
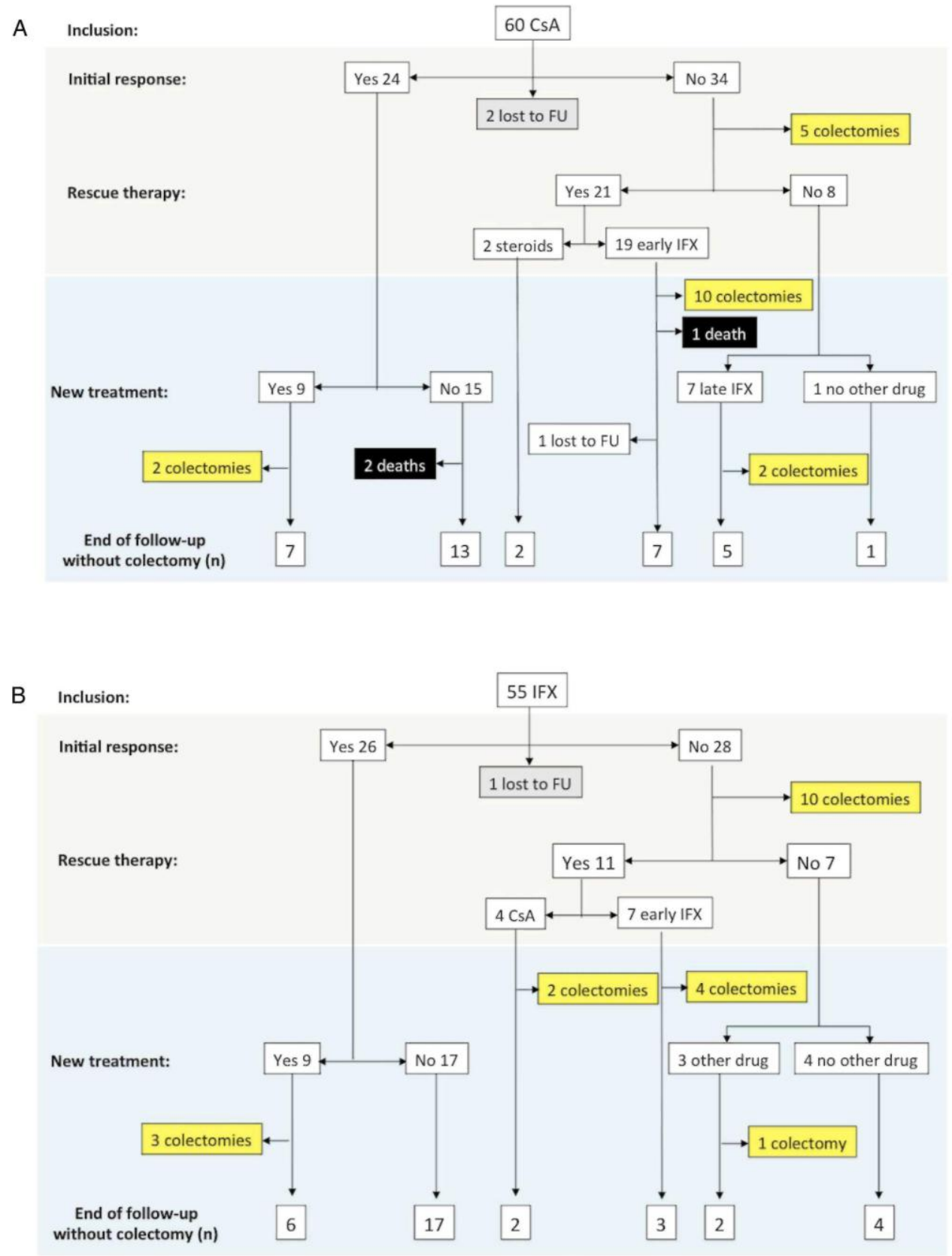
Among the 55 patients who started infliximab at inclusion (figure 1B), there were 27 failures, 26 successes, 1 lost to follow-up and 1 severe adverse event leading to treatment interruption (who underwent colectomy after 4 months) in the initial study. Among the 27 failures, 9 patients underwent directly colectomy after $0.7(0.2-2.2)$ months, 11 received rescue therapy (4 ciclosporin and 7 infliximab $-5 \mathrm{mg} / \mathrm{kg}$ in 3 and $10 \mathrm{mg} / \mathrm{kg}$ in 4 ) at 0.9 (0.62.0) months and 7 did not receive rescue therapy. Concerning the four patients who received ciclosporin, two underwent colectomy 0.3 and 0.9 months after rescue and two reached end of follow-up 25 and 61 months, the latter was subsequently treated by infliximab 50 months after rescue. Among the seven patients who received infliximab rescue therapy, four underwent colectomy after 0.2 to 3.3 months, and three reached end of follow-up 65 to 70 months later. Among the seven patients with infliximab failure who did not received rescue therapy, three had new systemic treatment 49 to 66 months after inclusion: one who received steroids and an experimental drug underwent colectomy 11 months later, one received steroids and infliximab and one received adalimumab reached end of follow-up 16 and 3 months after treatment. The four remaining patients had no new treatment and reached end of follow-up 59 to 92 months after inclusion. Among the 26 patients treated with no treatment failure at the end of the study period, various infliximab maintenance regimens were given (infliximab alone in 2, azathioprine alone in 7 and combotherapy in 17): 17 patients reached end of follow-up 73 (62-83) months after inclusion, 1 underwent colectomy 9 months after inclusion and 8 received new systemic treatment 24 (18-48) months after inclusion (2 underwent colectomy after 1 and 34 months after new treatment despite infliximab or adalimumab, 6 received various medical therapies - 1 infliximab, 2 steroids, 1 steroids then adalimumab, 1 adalimumab, 1 azathioprine - and were still followed up 29 (20-47) months later). Ten patients needed an infliximab intensification by shortening interval and/or increasing dose that was a failure in seven patients (four underwent colectomy, two switched to adalimumab and one was included in a trial) and successful in three.

\section{Death and colectomy}

Three patients died during the follow-up period: a male aged 66 years who received ciclosporin died from myocardial infarction 4 months after inclusion in the trial, a male aged 55 years treated successively with ciclosporin and infliximab died from pancreatic adenocarcinoma 21 months after inclusion and a female aged 69 years exposed to ciclosporin died from non-specified reasons 29 months after enrolment.

At follow-up update, 39 patients underwent colectomy: 19 treated initially with ciclosporin and 20 with infliximab. Colectomy-free survival rates at 1, 2 and 5 years according to treatment given at inclusion were, respectively, $70.9 \%$ (95\% CI $59.2 \%$ to $82.6 \%$ ), $65.3 \%$ (95\% CI $53.0 \%$ to $77.7 \%$ ) and $61.5 \%$ (95\% CI $48.7 \%$ to $74.2 \%$ ) in patients who received ciclosporin and $69.1 \%$ (95\% CI $56.9 \%$ to $81.3 \%$ ), $67.3 \%$ (95\% CI $54.9 \%$ to $79.7 \%$ ) and $65.1 \%$ (95\% CI 52.4\% to $77.8 \%$ ) in those who received infliximab (HR (95\% CI) $0.99(0.49$ to $1.98) ; \mathrm{p}=0.97$ ) (figure 2). Among the 39 patients who underwent colectomy, $31(79 \%)$ had pouch surgery-in three steps in 19 cases-3 $(8 \%)$ an ileorectal anastomosis and the remaining $5(13 \%)$ a permanent stoma. Ten $(26 \%)$ patients experienced postoperative complications within the 30 days after colectomy, including four infections, three bleedings and two occlusions and one cardiac failure.

\section{UC medical treatment}

Cumulative incidence of the first additional systemic treatment at 1,2 and 5 years according to treatment given at inclusion was, respectively, $52.6 \%$ (95\% CI $39.0 \%$ to $64.6 \%$ ), 58.3\% (95\% CI $44.3 \%$ to $70.0 \%$ ) and $62.3 \%$ (95\% CI $48.1 \%$ to $73.7 \%$ ) in patients who received ciclosporin and $20.0 \%$ (95\% CI $10.6 \%$ to $31.5 \%$ ), $27.3 \%$ (95\% CI $16.2 \%$ to $39.5 \%$ ) and $36.6 \%$ (95\% CI $23.9 \%$ to $49.3 \%$ ) in those who received infliximab (ARR (95\% CI) $0.547(0.350 \%$ to $0.854 \%) ; \mathrm{p}=0.011)$ (figure 3 ).

During the follow-up period, 33 patients treated first with ciclosporin and then received infliximab. Cumulative incidence of the first use of infliximab at 1,2 and 5 years in patients first treated with ciclosporin was, respectively, $45.7 \%$ (95\% CI $32.6 \%$ to $57.9 \%$ ), $53.3 \%$ (95\% CI 39.4\% to $65.3 \%$ ) and $57.1 \%$ (95\% CI $43.0 \%$ to $69.0 \%$ ) (figure 4). By contrast, four patients from the infliximab were treated with the converse sequence.

\section{New UC medical treatment}

When considering new UC therapies given during the follow-up period, we excluded those given at inclusion (ciclosporin/infliximab and azathioprine) in the initial study; this definition allows drug discontinuation and restart and/or drug optimisation. With this consideration, cumulative incidence of the first use of new UC systemic treatment or colectomy at 1, 2 and 5 years was, respectively, 52.6\% (95\% CI 39.0\% to 64.6\%), 58.3\% (95\% CI $44.3 \%$ to $70.0 \%$ ) and $62.3 \%$ (95\% CI $48.1 \%$ to $73.7 \%$ ) in patients who received ciclosporin and $9.1 \%$ 
(95\% CI $3.3 \%$ to $18.5 \%$ ), $12.7 \%$ (95\% CI $5.5 \%$ to $23.0 \%$ ) and $22.0 \%$ (95\% CI $12.0 \%$ to $33.8 \%$ ) in those who received infliximab (ARR $(95 \% \mathrm{CI}) 0.273$ (0.148 to 0.504$) ; \mathrm{p}<0.001)$ (figure 5).

Figure 2 Kaplan-Meier curves of colectomy-free survival according to treatment given at inclusion. CsA/Cys, ciclosporin; IFX, infliximab.

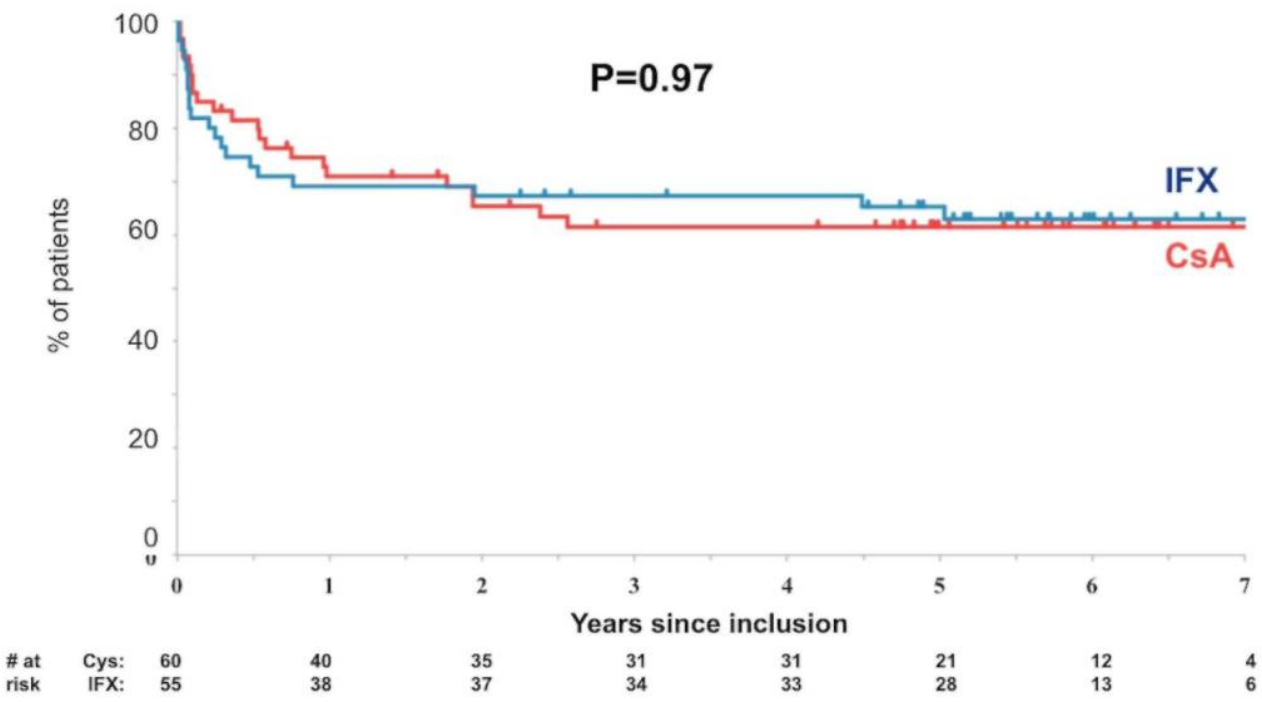

Figure 3 Cumulative incidence of the first use of any additional UC systemic treatment (steroids, ciclosporin, infliximab, azathioprine or other biologics) according to treatment given at inclusion. Full line: ciclosporin (CsA). Dotted line: infliximab (IFX).

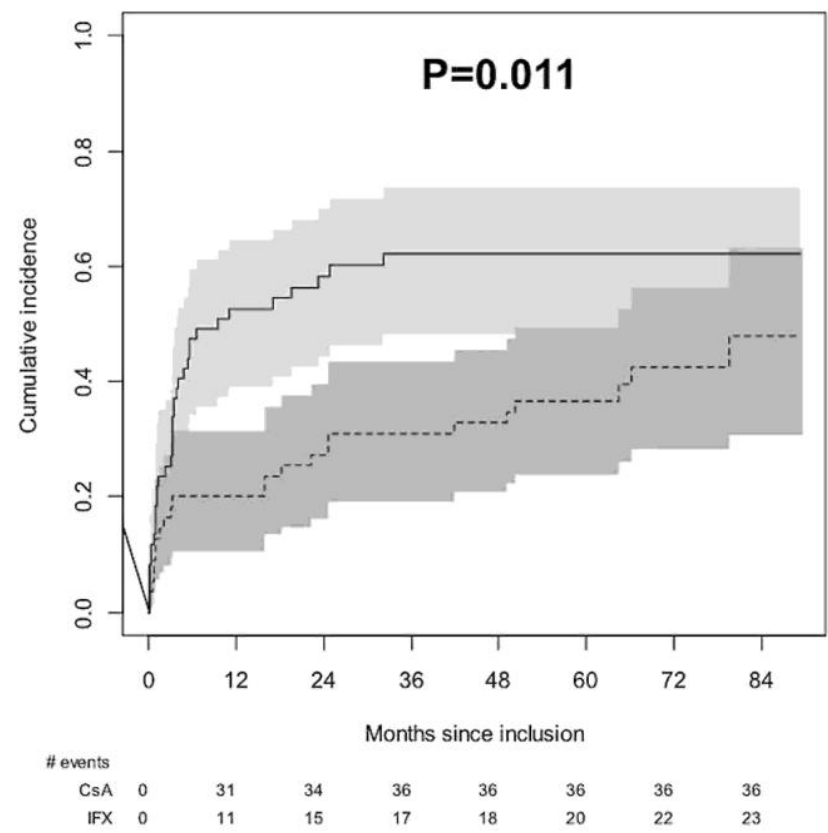

\section{DISCUSSION}

In a multicentre prospective cohort of patients with ASUC refractory to intravenous steroids who were randomly assigned for receiving ciclosporin or infliximab as second-line medical therapy, the long-term colectomy-free 
survival was independent from initial treatment received. However, a higher proportion of patients initially treated with ciclosporin required systemic subsequent therapies when compared with those who received infliximab first.

Both treatments have demonstrated their short-term efficacy as rescue therapies after steroid failure in patients with ASUC. Intravenous ciclosporin is rapidly and highly effective in this situation, with clinical response rates above $80 \%$ at 1 week in controlled trials. ${ }^{79}$ Nevertheless, this great efficacy is counterbalanced by safety concerns - such as opportunistic infections, renal dysfunction, high blood pressure or seizures — and is decreasing rapidly with time as nearly half of patients will relapse within 1 year despite maintenance therapy with thiopurines. ${ }^{14}$ In a cohort of 142 patients with ASUC who received intravenous ciclosporin before the antiTNF era, nearly all the patients treated experienced disease relapse and required surgery, with a 5-year colectomy rate of $69 \% .{ }^{15}$ Such an historical observation contrasts with more recent data with ciclosporin from the CONSTRUCT trial and this study showing death/colectomy rates of $48.1 \%$ at 3 years ${ }^{11}$ and $38.5 \%$ at 5 years, respectively. The more favourable outcome after ciclosporin treatment observed in our cohort may be related to the selection of patients who were azathioprine-naive and optimal candidates for a bridge strategy. It could also be explained by subsequent infliximab use, as observed within the first year in nearly half of the patients. Interestingly, 77\% of failures in the ciclosporin group occurred within the first year, and infliximab given in this situation was well tolerated and provided clinical benefit as approximately half of patients avoided colectomy at 5 years.

Figure 4 Cumulative incidence of the first infliximab use in the 60 patients treated with ciclosporin at inclusion.

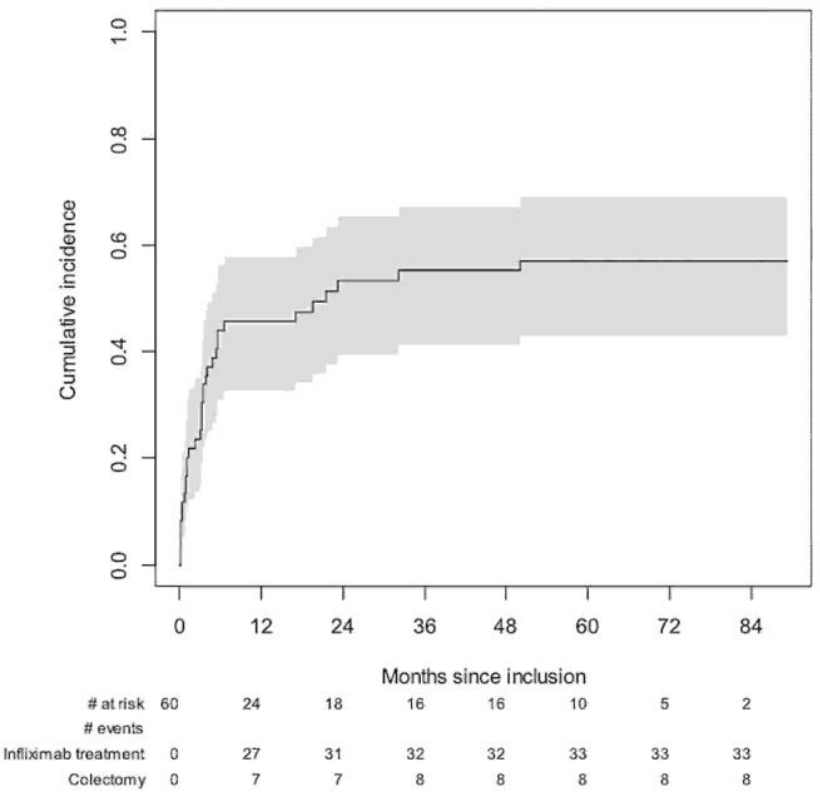

Long-term data on cohorts of inpatients with ASUC treated by infliximab as second-line medical therapy are scarce and usually mixing their results with a subgroup of patients having non-severe disease. In the CONSTRUCT trial, the 3-year colectomy rate in patients randomised for receiving infliximab was $40.7 \%,{ }^{11}$ comparable to the $34.9 \%$ observed at 5 years in the present cohort.

ASUC mortality rate remains high, around $1 \%-2 \%$ in the most recent series, mainly when colectomy has been delayed in elderly patients with comorbidities. ${ }^{3}$ Indeed, deaths were observed in $3 / 270(1 \%)$ patients in the CONSTRUCT trial, all of them treated with infliximab including two postoperative deaths, and in 3/115 (3\%) patients in the present cohort, all over 50 years and none related to the disease or the immunosuppression. 
Figure 5 Cumulative incidence of the first use of an additional UC systemic treatment other than those given at inclusion. Full line: ciclosporin (CsA). Dotted line: infliximab (IFX).

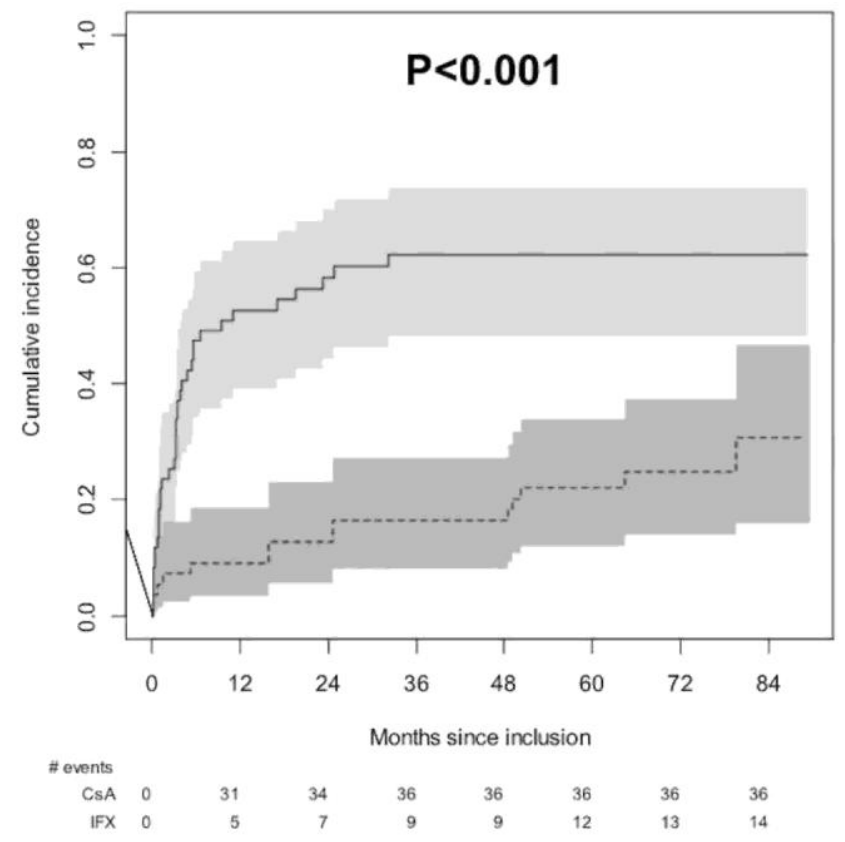

Within the last decade, IBD costs have dramatically increased with the spread of biologics use. In a recent Dutch study, anti-TNF are accounting for $31 \%$ of costs in UC. ${ }^{16}$ In several countries, direct costs are driving the choice between ciclosporin and infliximab in patients with ASUC. If so, and despite a longer duration of hospital stay at beginning, 3-month direct costs related to ciclosporin are significantly lower than with infliximab. ${ }^{17}$ Such a cost difference between both drugs has been confirmed by the CONSTRUCT trial. ${ }^{11}$

One of the strengths of this study is the initial randomisation in a well-defined population, providing treatment groups comparable at inclusion for baseline characteristic except treatment. This advantage may be slightly limited by the analysis according to treatment given instead of allocated, as two patients randomised into the infliximab arm received ciclosporin. The second limitation could be the long follow-up duration. Indeed, beyond the study period the protective effect of randomisation is decreasing with time as treatment decision is no more protocolised and may depend on physician and/or patient. Nevertheless, it should be noted that the clear-cut $p$ values for comparing rates for colectomy-free survival and incidence of the first use of new treatment guarantee our conclusions. Moreover, the follow-up duration of the present cohort seems appropriate for exploring the long-term outcome in patients with ASUC as its median duration was more than 5 years.

Overall, whatever the drug started as rescue therapy in patients with steroid-refractory ASUC, long-term colectomy rates are similar between ciclosporin and infliximab, both drugs having close efficacy and safety. Nearly half of patients first treated with ciclosporin will switch quickly to infliximab, within 1 year.

Accordingly, bridge therapy with ciclosporin to thiopurine was successful in the other half of patients. For daily practice, choice between both drugs should not only be guided by efficacy and safety criteria that are close, but should also take into account costs, administration route and patient's preference.

Acknowledgements The GETAID received unrestricted study grants from the Association Francois Aupetit.

Contributors DL: study concept and design; acguisition of data; analysis and interpretation of data; drafting of the manuscript; critical revision of the manuscript for important intellectual content; statistical analysis; obtained funding; technical or material support; study supervision. JYM: study concept and design; analysis and interpretation of data; drafting of the manuscript; critical revision of the manuscript for important intellectual content; statistical analysis; technical or material support; study supervision. JL: statistical analysis. AB, JB, MA, YB, JF, FZ, GS, LV, JM, AA, JC, ER, OD, ALS, MF, FC, GB, BC, XR, GVA, ME, MF, JPG, PM, SN, MDV 
and EL: acguisition of data and critical revision of the manuscript.

Funding Association Francois Aupetit.

Competing interests DL: consulting and/or lecture fees from Abbvie, Ferring, Janssen, MSD, Pfizer, Takeda. AB: consulting and/or lecture fees from Abbvie, Ferring, MSD, Pfizer, Takeda. MA: consulting and/or lecture fees from Janssen, MSD, Takeda. YB: consulting and/or lecture fees from Abbvie, Ferring, Hospira, Janssen, MSD, Pfizer, Takeda. JF: consulting and/or lecture fees from Abbvie, Ferring, MSD, Takeda. FZ: lecture fees for Abbvie, Takeda. GS: lecture fees from Abbvie, Ferring, MSD, Takeda. LV: lecture fees from Abbvie, Ferring, MSD, Hospira and Takeda and consulting fees from Abbvie. JM: lecture fees from Abbvie, Ferring, MSD, Takeda. AA: consulting and/or lecture fees from Abbvie, Ferring, MSD, Takeda. JC: consulting fees from Abbvie, Vifor. ER: consulting and/or lecture fees from Abbvie, MSD. OD: lecture fees from MSD. AL-S: lecture fees from Abbvie, MSD. MF: lecture fees from Abbvie, MSD, Takeda. GB: lecture fees from Abbvie, MSD, Takeda. BC: lecture fees from Abbvie, MSD. XR: consulting and/or lecture fees from Abbvie, Ferring, Janssen, MSD, Pfizer, Takeda, Theradiag. GvA: consulting and/or lecture fees and research support from MSD, Novartis. ME: consulting and/or lecture fees for MSD, Abbott, Shire Pharmaceuticals. JPG: consulting and/or lecture fees Abbvie, Janssen, Hospira, MSD, Pfizer, Takeda. PM: consulting and/or lecture fees from Abbvie, Ferring, MSD, Pfizer, Takeda. SN: consulting and/or lecture fees from Abbvie, Ferring, MSD, Takeda. MdV: consulting and/or lecture fees from Abbott, Ferring, MSD. EL: research grants from AstraZeneca, ScheringPlough; consulting and/or lecture fees from Abbott, Abbvie, AstraZeneca, Ferring, Schering-Plough, MSD, Chiesi, Menarini, Millenium, Mitsubishi Pharma, Nycomed, Falk, Takeda, UCB.

\section{Patient consent Obtained.}

Ethics approval The institutional review board at each centre approved the protocol, and all patients provided written informed consent.

Provenance and peer review Not commissioned; externally peer reviewed.

\section{REFERENCES}

1 Edwards FC, Truelove SC. The course and prognosis of ulcerative colitis. Gut 1963;4:299-315.

2 Dinesen LC, Walsh AJ, Protic MN, et al. The pattern and outcome of acute severe colitis. J Crohns Colitis 2010;4:431-7.

3 Lynch RW, Lowe D, Protheroe A, et al. Outcomes of rescue therapy in acute severe ulcerative colitis: data from the United Kingdom inflammatory bowel disease audit. Aliment Pharmacol Ther 2013;38:935-45.

4 Kaplan GG, McCarthy EP, Ayanian JZ, et al. Impact of hospital volume on postoperative morbidity and mortality following a colectomy for ulcerative colitis. Gastroenterology 2008;134:680-7.

5 Dignass A, Lindsay JO, Sturm A, et al. Second European evidence-based consensus on the diagnosis and management of ulcerative colitis part 2: current management. J Crohns Colitis 2012;6:991-1030.

6 Truelove SC, Witts LJ. Cortisone in ulcerative colitis; final report on a therapeutic trial. Br Med J 1955;2:1041-8.

7 Lichtiger S, Present DH, Kornbluth A, et al. Cydosporine in severe ulcerative colitis refractory to steroid therapy. $N$ Engl J Med 1994;330:1841-5.

8 Jarnerot G, Hertervig E, Friis-Liby I, et al. Infliximab as rescue therapy in severe to moderately severe ulcerative colitis: a randomized, placebo-controlled study. Gastroenterology 2005; 128:1805-11.

9 Van Assche G, D'Haens G, Noman M, ef al. Randomized, double-blind comparison of $4 \mathrm{mg} / \mathrm{kg}$ versus $2 \mathrm{mg} / \mathrm{kg}$ intravenous cydosporine in severe ulcerative colitis. Gastroenterology 2003; 125:1025-31.

10 Laharie D, Bourreille A, Branche J, et al. Cidosporine versus infliximab in acute severe colitis refractory to intravenous steroids: a randomized study. Lancet 2012;380:1909-15.

11 Williams JG, Alam MF, Alrubaiy L, et al. Comparison Of iNfliximab and cidosporin in STeroid Resistant Ulcerative Colitis: pragmatic randomised Trial and economic evaluation (CONSTRUCT). Health Technol Assess 2016;20:1-320.

12 Kalbfleisch, JD, Prentice RL. The statistical analysis of failure time data. New York: Wiley, 1980.

13 Gerds TA, Scheike TH, Andersen PK. Absolute risk regression for competing risks: interpretation, link functions and prediction. 
Published in : Gut (2017)

Status : Postprint (Author's version)

Statist Med 2012;31: 3921-30.

14 Seah D, De Cruz P. Review article: the practical management of acute severe ulcerative colitis. Review article: the practical management of acute severe ulcerative colitis. Aliment Pharmacol Ther 2016;43:482-513.

15 Moskovitz DN, Van Assche G, Maenhout B, et al. Incidence of colectomy during long-term follow-up after cydosporine-induced remission of severe ulcerative colitis. Clin Gastroenterol Hepatol 2006;4:760-5

16 van der Valk ME, Mangen MJ, Leenders M, et al. Healthcare costs of inflammatory bowel disease have shifted from hospitalisation and surgery towards anti-TNF $\alpha$ therapy: results from the COIN study. Gut 2014;63:72-9.

17 Löwenberg M, Duijvis NW, Ponsioen C, et al. Length of hospital stay and associated hospital costs with infliximab versus cydosporine in severe ulcerative colitis. Eur J Gastroenterol Hepatol 2014;26:1240-6.

18 Satsangi J, Silverberg MS, Vermeire S, et al. The Montreal classification of inflammatory bowel disease: controversies, consensus, and implications. Gut 2006;55:749-53. 